\title{
A bright, high rotation-measure FRB that skewers the M33 halo
}

Liam Connor ${ }^{1,2}$, Joeri van Leeuwen ${ }^{2,1}$, L. C. Oostrum ${ }^{2,1}$, E. Petroff ${ }^{1,3}$, Yogesh Maan², E. A. K. Adams ${ }^{2,4}$, J. J. Attema ${ }^{5}$, J. E. Bast ${ }^{2}$, O. M. Boersma ${ }^{1,2}$, H. Dénes ${ }^{2}$, D. W. Gardenier ${ }^{2,1}$, J. E. Hargreaves ${ }^{2}$, E. Kooistra ${ }^{2}$, I. Pastor-Marazuela ${ }^{1,2}$, R. Schulz ${ }^{2}$, A. Sclocco ${ }^{4}$, R. Smits ${ }^{2}$, S. M. Straal ${ }^{6,7}$, D. van der Schuur ${ }^{2}$, Dany Vohl², B. Adebahr ${ }^{8}$, W. J. G. de Blok $2,9,4$, W. A. van Cappellen ${ }^{2}$, A. H. W. M. Coolen ${ }^{2}$, S. Damstra ${ }^{2}$, G. N. J. van Diepen ${ }^{2}$, B. S. Frank ${ }^{10,9}$, K. M. Hess ${ }^{2,4}$, B. Hut ${ }^{2}$, A. Kutkin ${ }^{2,11}$, G. Marcel Loose ${ }^{2}$, D. M. Lucero ${ }^{12}$, Á Mika², V. A. Moss ${ }^{13,14,2}$, H Mulder ${ }^{2}$, T. A. Oosterloo ${ }^{2,4}$, M Ruiter ${ }^{2,2}$, H. Vedantham², N. J. Vermaas ${ }^{2}$, S. J. Wijnholds ${ }^{2}$, J. Ziemke 2,15

\footnotetext{
1 Anton Pannekoek Institute, University of Amsterdam, PO Box 94249, 1090 GE Amsterdam, The Netherlands

${ }^{2}$ ASTRON, the Netherlands Institute for Radio Astronomy, Oude Hoogeveensedijk 4, 7991 PD Dwingeloo, The Netherlands

3 Veni Fellow

${ }^{4}$ Kapteyn Astronomical Institute, PO Box 800, 9700 AV Groningen, The Netherlands

5 Netherlands eScience Center, Science Park 140, 1098 XG, Amsterdam, The Netherlands

6 NYU Abu Dhabi, PO Box 129188, Abu Dhabi, United Arab Emirates

7 Center for Astro, Particle, and Planetary Physics (CAP $)$, NYU Abu Dhabi, PO Box 129188, Abu Dhabi, United Arab Emirates

8 Astronomisches Institut der Ruhr-Universität Bochum (AIRUB), Universitätsstrasse 150, 44780 Bochum, Germany

9 Dept. of Astronomy, Univ. of Cape Town, Private Bag X3, Rondebosch 7701, South Africa

10 South African Radio Astronomy Observatory (SARAO), 2 Fir Street, Observatory, 7925, South Africa

11 Astro Space Center of Lebedev Physical Institute, Profsoyuznaya Str. 84/32, 117997 Moscow, Russia

12 Department of Physics, Virginia Polytechnic Institute and State University, 50 West Campus Drive, Blacksburg, VA 24061, USA

13 CSIRO Astronomy and Space Science, Australia Telescope National Facility, PO Box 76, Epping NSW 1710, Australia

${ }^{14}$ Sydney Institute for Astronomy, School of Physics, University of Sydney, Sydney, New South Wales 2006, Australia

15 Rijksuniversiteit Groningen Center for Information Technology, P.O. Box 11044, 9700 CA Groningen, the Netherlands
}

\begin{abstract}
We report the detection of a bright fast radio burst, FRB 191108, with Apertif on the Westerbork Synthesis Radio Telescope (WSRT). The interferometer allows us to localise the FRB to a narrow $5^{\prime \prime} \times 7^{\prime}$ ellipse by employing both multibeam information within the Apertif phased-array feed (PAF) beam pattern, and across different tiedarray beams. The resulting sight line passes close to Local Group galaxy M33, with an impact parameter of only $18 \mathrm{kpc}$ with respect to the core. It also traverses the much larger circumgalactic medium of M31, the Andromeda Galaxy. We find that the shared plasma of the Local Group galaxies could contribute $\sim 10 \%$ of its dispersion measure of $588 \mathrm{pc} \mathrm{cm}^{-3}$. FRB 191108 has a Faraday rotation measure of $+474 \pm 3 \mathrm{rad} \mathrm{m}^{-2}$, which is too large to be explained by either the Milky Way or the intergalactic medium. Based on the more moderate RMs of other extragalactic sources that traverse the halo of M33, we conclude that the dense magnetised plasma resides in the host galaxy. The FRB exhibits frequency structure on two scales, one that is consistent with quenched Galactic scintillation and broader spectral structure with $\Delta v \approx 40 \mathrm{MHz}$. If the latter is due to scattering in the shared M33/M31 CGM, our results constrain the Local Group plasma environment. We found no accompanying persistent radio sources in the Apertif imaging survey data.
\end{abstract}

Key words: fast radio bursts - observation - instrumentation 


\section{INTRODUCTION}

Fast radio bursts (FRBs) are extragalactic radio pulses, of which approximately 110 have been discovered to date (Lorimer et al. 2007; Petroff et al. 2016). They are short duration ( $\mu \mathrm{s}-\mathrm{ms})$, bright (0.01-100 Jy peak flux density), highly dispersed, and relatively common $\left(\sim 10^{3} \mathrm{sky}^{-1} \mathrm{day}^{-1}\right.$ above 1 Jy; Cordes \& Chatterjee 2019; Petroff et al. 2019). The most pressing questions in FRB science fall into two broad categories: What causes these mysterious bursts? And, how can they be put to use?

In the former class of questions, significant progress has been made in the past several years. A subset of FRBs has been found to repeat, the first of which was the Arecibodiscovered FRB 121102 (Spitler et al. 2014, 2016). Eighteen repeaters have been detected with the Canadian Hydrogen Intensity Mapping Experiment (CHIME) (CHIME/FRB Collaboration et al. 2019b,c; Fonseca et al. 2020) as well as one from ASKAP (Kumar et al. 2019). It is still unclear if the sources that have not been seen to repeat are of a distinct class of once-off events, or if their repetition statistics (rate, temporal clustering, luminosity function, etc.) are such that they are difficult to detect more than once with most telescopes (e.g. Kumar et al. 2019). Real-time arcsecond localisation has allowed for host galaxy identifications, shedding light on the variety of galaxies in which FRBs reside (Bannister et al. 2019; Ravi et al. 2019). Very-longbaseline interferometry (VLBI) follow up of repeating FRBs has provided milliarcsecond localisation, which has been essential in understanding the nearby progenitor environment (Marcote et al. 2017; Chatterjee et al. 2017; Tendulkar et al. 2017; Bassa et al. 2017; Michilli et al. 2018; Marcote et al. 2020).

In the FRB applications category, the theoretical proposals that have been put forward range from intergalactic medium (IGM) and circumgalactic medium (CGM) studies (McQuinn 2014; Prochaska \& Zheng 2019; Vedantham \& Phinney 2019), to gravitational lensing (Muñoz et al. 2016; Eichler 2017) and cosmology (Walters et al. 2018). Recently, progress has been made in putting such proposals into practice (Ravi et al. 2016; Prochaska et al. 2019).

In this paper we report the detection of FRB 191108 with the Apertif Radio Transient System (ARTS) on the Westerbork Synthesis Radio Telescope (WSRT). This source has a Faraday Rotation Measure $\mathrm{RM}=+474 \pm 3 \mathrm{rad} \mathrm{m}^{-2}$, which is an order of magnitude larger than the expected Galactic and IGM contributions. It also passes through the halo of Local Group galaxy M33 (The Triangulum Galaxy) with a best-fit impact parameter of just $18 \mathrm{kpc}$. The M33 halo is embedded in the much-larger galactic halo of M31 (The Andromeda Galaxy), which we expect to also impact the propagation of the pulse. In Sect. 2 we briefly describe the discovery pipeline. We present the burst discovery and localization efforts in Sect. 3, and discuss rotation measure and repetition constraints in Sect. 4 and conclude in Sect. 5 .

\section{ARTS PIPELINE}

The Apertif Radio Transient System (ARTS) searches for radio pulses using ten 25-m dishes of the WSRT equipped with the new Apertif phased array feeds (PAFs; Oosterloo et al. 2010; Adams \& van Leeuwen 2019). While a full description of ARTS is provided in van Leeuwen et al. (2020), we highlight a number of relevant features below.

For the real-time FRB search, we beamform the dipoles in each of the PAFs to produce 40 voltage 'compound beams' (CBs) with $300 \mathrm{MHz}$ of bandwidth centered on a radio frequency of $1370 \mathrm{MHz}$. This is done at each dish. The compound beams are next further beamformed in firmware across the East-West array to create 12 tied-array beams (TABs) per compound beam, out of which we generate Stokes I, Q, U, and V data-streams at $81.92 \mu$ s and $195 \mathrm{kHz}$ time and frequency resolution. As the fractional bandwidth of Apertif is high, $\sim 0.2$, the TABs must be recombined in frequency to produce 'synthesised beams' (SBs). A synthesised beam points in the same direction across the $300 \mathrm{MHz}$ band, which is not true of a TAB. An overview of this hierarchical beamforming is provided in van Leeuwen et al. (2020). In total, 71 synthesised beams are formed per compound beam, which span the full primary beam field of view (FoV) of $\sim 0.23 \mathrm{deg}^{2}$. The full 40-compound-beam PAF has a FoV of roughly $9 \mathrm{deg}^{2}$. The total 2840 Stokes I synthesized beams are then searched in real time by our single-pulse search software AMBER $^{1}$ (Sclocco et al. 2016, 2020), which runs on a dedicated 40-node graphics processing unit (GPU) computing cluster at the WSRT site. Data post-processing is handled by the Data Analysis of Real-time Candidates from the Apertif Radio Transient System (DARC ARTS ${ }^{2}$; Oostrum 2020a) pipeline. Raw candidates are clustered in dispersion measure (DM), time, pulse width, and beam number; and then sent to a machine learning classifier which assigns a probability of the candidate being a true FRB (Connor \& van Leeuwen 2018). While Stokes I data is always written to filterbank files on disk, the buffered Stokes Q, U, and $\mathrm{V}$ data are only saved if AMBER identifies a candidate with a total duration $<10 \mathrm{~ms}$, a signal-to-noise ratio ( $\mathrm{S} / \mathrm{N}$ hereafter) greater than 10, and a DM more than $20 \%$ larger than the predicted value along the line of sight from the YMW16 electron density model (Yao et al. 2017).

\section{RESULTS}

FRB 191108 was detected in three compound beams, at solar system barycentric UTC 19:48:50.240. The discovery DM was $588 \mathrm{pc} \mathrm{cm}^{-3}$. Fig. 1 shows the dynamic spectrum of the dispersed pulse as well as the dedispersed pulse profile. The maximum $\mathrm{S} / \mathrm{N}$ from the real-time detection was 60 in compound beam 21 (see Fig. 3) and our machine learning classifier assigned a probability of $>99.9 \%$ of it being a real transient (Connor \& van Leeuwen 2018). The AMBER detection triggered a dump of the full-Stokes data, allowing us to analyse the polarisation properties of the burst.

\subsection{Polarisation properties}

FRB 191108 was measured to be roughly $70 \%$ linearly polarised and $\leq 10 \%$ circularly polarised. It was found to have a rotation measure $(\mathrm{RM})$ of $+474 \pm 3 \mathrm{rad} \mathrm{m}^{-2}$. The best-fit $\mathrm{RM}$

\footnotetext{
1 https://github.com/AA-ALERT/AMBER

2 https://github.com/loostrum/darc
} 


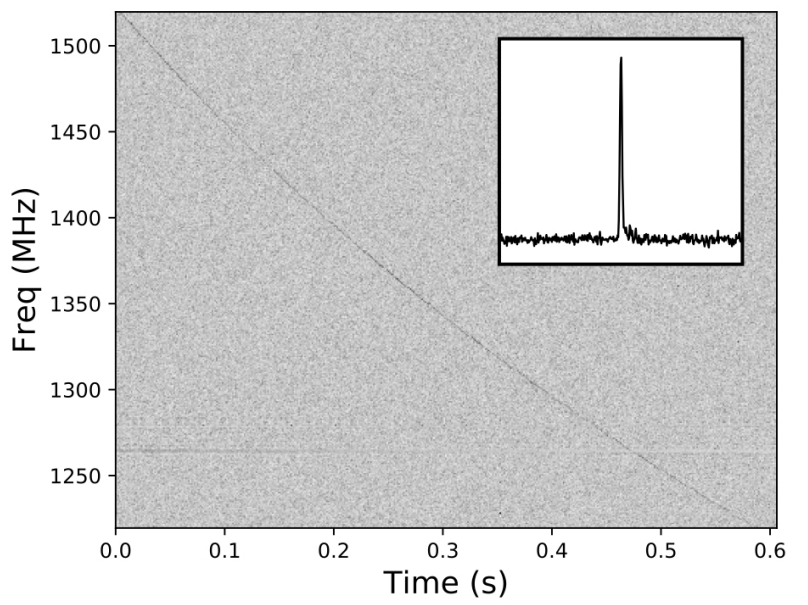

Figure 1: The dispersed dynamic spectrum of FRB 191108 across the ARTS observing bandwidth, and the dedispersed and frequency-averaged pulse profile for $30 \mathrm{~ms}$ of data (inset). The dynamic spectrum has been bandpass corrected and median subtracted, but not RFI cleaned. It is has been binned down to $0.82 \mathrm{~ms}$ time resolution with $0.78 \mathrm{MHz}$ frequency channels.

was obtained by applying a linear least squares fit to position angle (PA) as a function of wavelength squared. The sign was determined by verifying that the Crab pulsar had an $\mathrm{RM}$ of $-43 \mathrm{rad} \mathrm{m}^{-2}$ during an observation the same day.

Both bandpass calibration and polarisation calibration were done using $3 \mathrm{C} 286$, a standard calibrator source, which is known to have very little circular polarisation. We treat the Stokes V value as an upper limit because of uncertainty in the polarisation calibration procedure. 3C286 was observed in the same compound beam as the FRB, but it was observed in the central TAB, where leakage is expected to be minimised. FRB 191108 was found in synthesised beam number 37, which is a linear combination of non-central TABs. That synthesised beam may have slightly different leakage properties than the central TAB, which will be better quantified as the system is further calibrated. From the 3C286 on/off observation, we solved for a single phase in each down-channelised frequency channel, knowing that the complex $X Y$ correlation ought to be purely real if Stokes $\mathrm{V}$ is zero. We verified that the polarisation calibration solution agreed with a different method that used the FRB itself, which separated the component of $\operatorname{Im}\{X Y\}$ that varies with $\lambda^{2}$ from that which does not, since Stokes V should not exhibit Faraday rotation under most circumstances. Fortunately, the polarisation rotation does not vary with parallactic angle on Westerbork data, as the dishes are on equatorial mounts. Thus, differences in hour angle between the two observations have no influence. Still, it is possible that the calibration solution is sufficiently different between TABs and synthesised beams that the observed $13 \%$ circular polarisation is spurious. Fortunately, Faraday rotation is robust against uncertainty in the polarisation calibration solution, because it is difficult to mimic a rotation in the $\mathrm{Q} / \mathrm{U}$ plane that is sinusoidal in $\lambda^{2}$. We are confident in the reported value of the rotation measure $(\mathrm{RM})$.

Cho et al. (2020) found that FRB 181112 exhibited changes in its polarisation PA both within and between subbursts. We see no evidence of a swing in the PA across the pulse. FRB 121102 was also found to have a flat polarisation PA (Michilli et al. 2018; Gajjar et al. 2018; Hessels et al. 2019), as was FRB 180916.J0158+65 (known as R3; CHIME/FRB Collaboration et al. 2019c). This is in contrast to many pulsars and it may have interesting implications for FRB emission mechanisms. In our case, however, the flat PA may be instrumental. While the true PA could be flat across the pulse like previous FRBs, the intrinsic width of FRB 191108 is temporally unresolved, meaning any swing in the polarisation PA is unobservable; the apparent flat PA across the pulse is the time-averaged angle of the true pulse. This can lead to depolarisation, because coarse temporal sampling and intra-channel dispersion effectively add linear-polarisation vectors across the pulse that may point in different directions. The depolarisation fraction is

$f_{\text {depol }}(\Delta \theta)=1-\cos (\Delta \theta / 2)$.

Here, $\Delta \theta$ is the PA change across the pulse in radians. Since we observe $\sim 70 \%$ of the FRB emission to be linearly polarised, the true pulse must be at least as polarised and its $\Delta \theta$ cannot be greater than $\sim 90^{\circ}$. It is possible that FRB 191108 and other temporally-smeared FRBs with moderate polarisation fractions have higher intrinsic polarisations than inferred.

\subsection{Localisation}

By combining multibeam information from the 40 overlapping compound beams ( $\mathrm{CBs}$ ) in a PAF, with the interferometric information contained in the TABs and synthesised beams (SBs), Apertif can achieve a theoretical localisation region of

$\Omega \approx \frac{30^{\prime \prime}}{S / N} \times \frac{30^{\prime}}{S / N}$

although in practice this will depend on the accuracy of our beam-shape models. In order to localise FRB 191108, we first need to obtain the $\mathrm{S} / \mathrm{N}$ of the burst in each $\mathrm{SB}$. The FRB was initially detected in two neighbouring compound beams, with the highest $\mathrm{S} / \mathrm{N}$ in $\mathrm{CB} 21$ (see Fig. 3). Using the postdetection optimised DM and timestamp, we measure the $\mathrm{S} / \mathrm{N}$ of the burst in all SBs of CB 21 and the ones surrounding it. Using a $\mathrm{S} / \mathrm{N}$ threshold of 8 , the FRB was detected in CBs 15, 21, and 22, across a total of 48 SBs. The highest S/N was 103 in SB 37 of CB 21 (hereafter the reference beam).

We create a model of the Apertif beam pattern assuming a Gaussian primary beam pattern for each compound beam, with a half-power width of $36.3^{\prime}$ at $1370 \mathrm{MHz}$. Each $\mathrm{CB}$ is then scaled using the system-equivalent flux density measured for each $\mathrm{CB}$ determined from a drift scan of calibrator source $3 \mathrm{C} 48$. Defining a grid of $40^{\prime} \times 40^{\prime}$ with a resolution of 1 " centered on CB 21, we generate the TAB response of the 8 equidistant WSRT dishes across this grid and recombine these across frequency into $71 \mathrm{SBs}$ per CB. The SBs are integrated across frequency, assuming a flat spectral index. The model is then scaled to the model of the reference beam, resulting in a prediction of the $\mathrm{S} / \mathrm{N}$ ratio between each $\mathrm{SB}$ and the reference beam. 


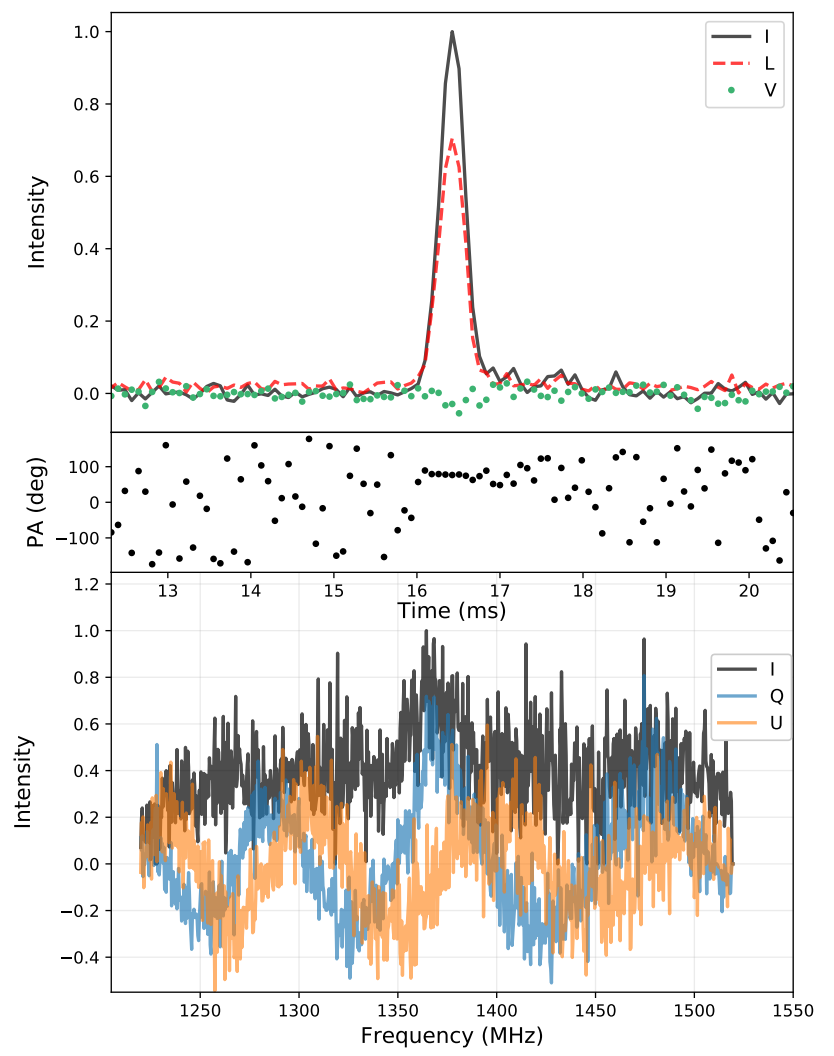

Figure 2: The measured polarisation properties of FRB 191108. The top panel shows the frequency-averaged pulse profiles after correcting for Faraday rotation in total intensity, I, linear polarisation, $\mathrm{L} \equiv \sqrt{\mathrm{Q}^{2}+\mathrm{U}^{2}}$, and circular polarisation, $\mathrm{V}$. The middle panel shows a flat PA across the pulse, which could be intrinsic or due to depolarisation, as the true FRB width is temporally unresolved. The bottom panel shows the band-pass corrected frequency spectrum, as well as the Faraday-rotated Stokes Q and U. The best fit $\mathrm{RM}$ is $+474 \pm 3 \mathrm{rad} \mathrm{m}^{-2}$.

Next, we calculate the $\chi^{2}$ statistic at each grid point. For SBs without a detection, we only include points where the modelled $\mathrm{S} / \mathrm{N}$ is above the detection threshold and use the $\mathrm{S} / \mathrm{N}$ threshold in place of the observed S/N. A $90 \%$ confidence region is derived from $\Delta \chi^{2}$ values using the theoretical conversion between confidence level and $\Delta \chi^{2}$. The localisation method has been verified using multi-beam detections of giant pulses from the Crab pulsar and single pulses from PSR J0528+2200, also in CB 21.

Our method is similar to that employed by CHIME (CHIME/FRB Collaboration et al. 2019b). The Bayesian approach taken by Bannister et al. (2017) for ASKAP is more elaborate, allowing for errors in beam size, sensitivity, and position. However, we note that ASKAP was operating in fly's eye mode. This limits the resolution of a single beam. In contrast, the Apertif data are coherently beamformed across the array, leading to many narrower, higherresolution beams. This limits the impact of uncertainty in the compound beam positions, as the direction of peak sensitivity of the resulting TABs is dominated by the phase

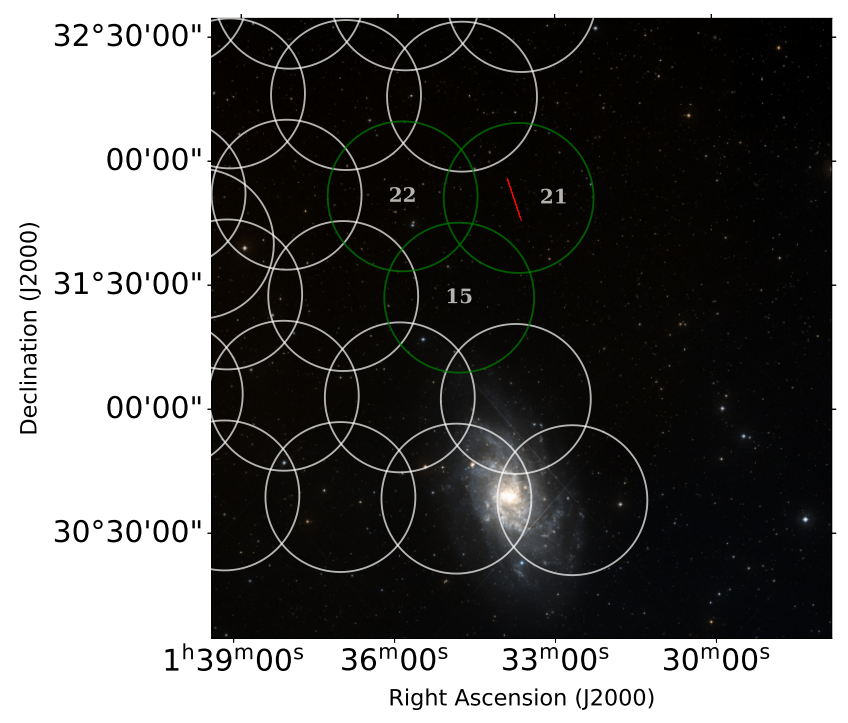

Figure 3: The localisation region of FRB 191108. The compound beams at $1370 \mathrm{MHz}$ are shown in white (nondetection) and green (detection, with circle opacity in proportion to $\mathrm{S} / \mathrm{N}$ ). The best-fit location is shown with a blue cross. The $90 \%$ confidence localisation area is an elongated ellipse, but it is represented in this figure by a red line due to the ellipse's large aspect ratio. The galaxy near the bottom of the figure is M33, which is $1.20 \pm 0.05^{\circ}$ from the location of the FRB. Background image from the Sloan Digital Sky Survey (SDSS; York et al. 2000).

offsets between the dishes. Further improvements to the determination of Apertif confidence regions, based on several pulsar observations, are in progress (Oostrum 2020b). The localisation code is available online ${ }^{3}$.

The final derived $90 \%$ confidence region is shown in Fig. 3. The best-fit position (J2000) corresponds to $\mathrm{RA}=01: 33: 47$, Dec $=+31: 51: 30$. The error ellipse has a semimajor axis of $3.5^{\prime}$ and a semi-minor axis of $2.5^{\prime \prime}$, with a position angle of $19.5^{\circ}$ East of North. The FRB is localised to a region $1.20 \pm 0.05^{\circ}$ from the core of Local Group galaxy M33. The localisation solid angle of approximately 2100 square arcseconds (90\% confidence) is too large to unambiguously identify a host galaxy associated with the FRB, even if the $\mathrm{DM} / z$ relation is to be trusted and utilised (Eftekhari \& Berger 2017). However, as we discuss in Sect. 4.2, if FRB 191108 is found to repeat and is detected at a different parallactic angle, we will achieve $\sim$ arcsecond localisation in both directions because the TABs will be at a different position angle on the sky.

\subsubsection{Apertif continuum survey $\&$ radio counterpart}

We have searched for a persistent radio source associated with FRB 191108 in continuum images from the Apertif imaging surveys (Hess et al. 2020 ${ }^{4}$ ). The mosaic in Fig. 4 is a combination of 31 compound beams from two survey pointings (191010042 and 191209026) which overlap around

3 https://github.com/loostrum/arts_localisation 
the localisation region. The continuum images for the mosaic were made using the top $150 \mathrm{MHz}$ of the Apertif imaging band (1280-1430 MHz). The mosaic covers $\sim 9 \mathrm{deg}^{2}$ and M33 can be seen in the bottom half of the map. We did not find anything within the localisation error region above $5 \sigma$ at $71 \mu \mathrm{Jy}$ root mean square noise.

Radio point sources have a lower on-sky density than faint optical galaxies, which decreases the probability of chance spatial coincidence and relaxes the localisation requirements for radio counterparts (Eftekhari et al. 2018). The persistent radio source associated with FRB 121102 was roughly $200 \mu \mathrm{Jy}$ at $z \approx 0.2$ at $1 \mathrm{GHz}$ (Chatterjee et al. 2017), meaning we could have detected an equivalent nebula above $3 \sigma$ if FRB 191108 were at the same distance as FRB 121102. This is more nearby than the maximum redshift implied by the extragalactic DM of FRB 191108, which is $z \approx 0.52$ (see Sect. 3.4.1). Therefore, the host-galaxy ISM or the dense magnetised plasma contributing to the RM of the FRB would need to contribute significant DM in order for us to detect a persistent source similar to the one associated with FRB 121102. This is not implausible: Using the same Galactic halo modelling and $\mathrm{DM} / z$ relation employed in this paper, the extragalactic DM of FRB 121102 implies a redshift that is $60 \%$ larger than the known value of its host galaxy. The Galactic center magnetar, PSR J1745-2900, is both strongly Faraday rotated $\left(\mathrm{RM} \approx 7 \times 10^{4} \mathrm{rad} \mathrm{m}^{-2}\right)$ and dispersed $\left(\mathrm{DM} \approx 1780 \mathrm{pc} \mathrm{cm}^{-3}\right.$ ) near to the source, which would make it seem very distant if it were bright enough to be seen by an extragalactic observer (Eatough et al. 2013). Nonetheless, we note that of the five unambiguously localised FRBs, no source has a host-galaxy DM that is known to be significantly more than half its extragalactic DM (Tendulkar et al. 2017; Bannister et al. 2019; Prochaska et al. 2019; Ravi et al. 2019; Marcote et al. 2020). More host-galaxy localisations will be needed to determine how common it is for FRBs to be strongly dispersed locally.

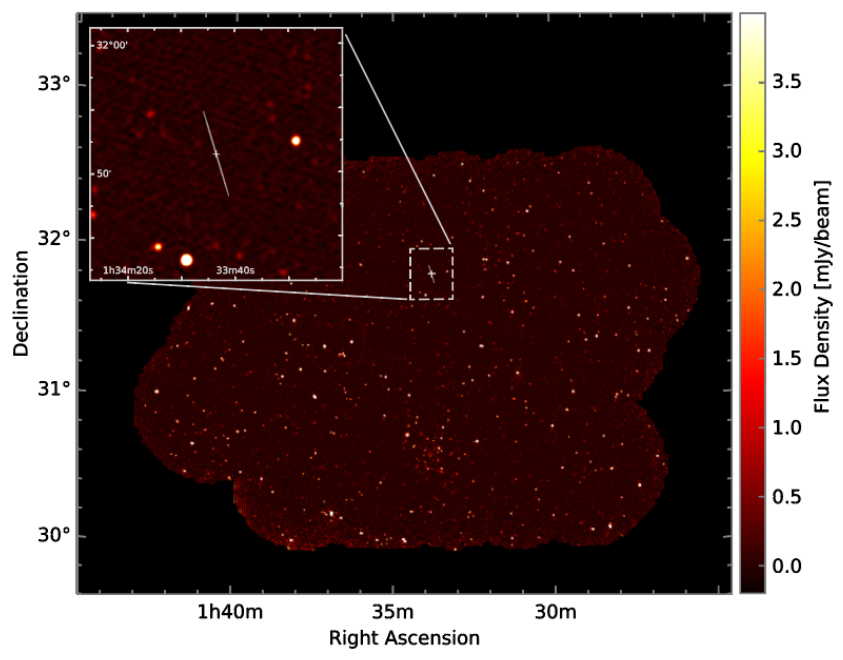

Figure 4: A mosaic from the Apertif imaging surveys combining 30 compound beams from two adjacent pointings around the localisation region. The mosaic has a synthesized beam of $25 \operatorname{arcsec} \times 25$ arcsec. In the FRB localisation region, marked by the white ellipse, no persistent radio counterpart brighter than $\sim 350 \mu \mathrm{Jy}$ ( $5 \sigma$ limit) was found.
If there were a radio source associated with M33 at $840 \mathrm{kpc}$, we can set an upper limit on its luminosity of $v L_{v}<8.5 \times 10^{31} \mathrm{erg} \mathrm{s}^{-1}$. At $1400 \mathrm{MHz}$, many supernova remnants (Chomiuk \& Wilcots 2009) and HII regions (Paladini et al. 2009) would have been detectable if they were at the same distance as M33. M33 is known to have RGB stars stretching $\sim 2^{\circ}$ north of the core, nearly three times the radius of the classical disk (McConnachie et al. 2009, 2010), due to past interactions with M31. The northern part of M33 also has many HII regions (Relano et al. 2013), but most are within $10 \mathrm{kpc}$ of the core (30 arcminutes below FRB 191108). Therefore, even though it is plausible that there would be stellar structure or star formation at the location of FRB 191108, we do not find evidence for a strong Faraday rotating plasma associated with M33. These facts, along with the arguments presented in Sect. 4.1, suggest the FRB RM arises in its host galaxy.

\subsection{Time \& frequency structure}

We do not find evidence of temporal scattering in FRB 191108 above $\sim 80 \mu$ s. Even though visually there appears to be slightly more power after the main peak of the FRB pulse profile than before it, the detected pulse width is consistent with intra-channel dispersion smearing and the sampling time of our instrument. We have also fit pulse width as a function of frequency and found the data to prefer dispersion smearing over scattering. The latter would result in a $\tau \propto v^{-4}$ relationship for a single-screen, whereas instrumental smearing between channels causes the width to scale as $v^{-3}$, assuming dispersion smearing is larger than sampling time. We find the best-fit $\tau(v)$ power-law to be -2.9 , implying that the pulse is temporally unresolved even at $275 \mu \mathrm{s}$. We also compared our pulse with simulation codes simpulse ${ }^{5}$ and injectfrb ${ }^{6}$, which generate realistically smeared FRBs and account for finite channelisation and temporal sampling. We simulated bursts with the same DM but varying intrinsic widths, assuming the same time and frequency resolution as ARTS, and fit their "observed" widths with the same pipeline that was used for the FRB. We found that the intrinsic width of FRB 191108, and any scatter-broadening, must be $\lesssim 80 \mu$ s.

In the top panel of Fig. 2, there is excess power after the primary pulse, and between 17 and $19 \mathrm{~ms}$ the PA appears non-random and consistent with the PA of the main pulse. Indeed, when the primary pulse is masked out, we find a $7.5 \sigma$ pulse whose best-fit width is $1 \mathrm{~ms}$. This broader, weaker sub-pulse after the bright, narrow main pulse has been seen in other FRBs, for example the repeating FRB 180916.J0158+65 (see pulse $d$ in Fig. 1 from Marcote et al. 2020) as well as the first repeater, FRB 121102 (see pulse $a$ in Fig. 1 from Michilli et al. 2018).

As argued by Connor (2019), the observed widths of many FRBs are close to the instrumental smearing timescale, i.e. $\sim \sqrt{\tau_{\mathrm{DM}}^{2}+t_{\text {samp }}^{2}}$, indicating that there may exist large numbers of narrow bursts that are missed by current search backends. When FRBs are coherently dedispersed or observed with high time/freq resolution, structure is often

5 https://github.com/kmsmith137/simpulse
6 https://github.com/liamconnor/injectfrb 
revealed on tens of microseconds timescales (Ravi et al. 2016; Farah et al. 2018; Hessels et al. 2019). FRB 191108 may therefore be an example of this population of narrow FRBs that are often missed without high time and frequency resolution backends - something Apertif is fortunate to have.

A least-squares power-law fit was applied to the Stokes I frequency spectrum of the FRB, yielding a power-law index of $-1.6 \pm 0.5$. But like other fast radio bursts, FRB 191108 is not well described by a power law. In the center and top of the band there is a factor of $\sim 2$ of excess power (see the bottom panel of Fig. 2). Our constraint on the scatterbroadening implies a lower limit on the Galactic scintillation originated decorrelation bandwidth to be a few $\mathrm{kHz}$. However, as argued in Section 3.4.2, the observed frequency modulation, with characteristic bandwidth of the order of $40 \mathrm{MHz}$, is unlikely to be due to scintillation. Such bandedness has been seen in more extreme cases by ASKAP (Shannon et al. 2018) and CHIME (CHIME/FRB Collaboration et al. 2019a), as well as in FRB 121102 (Hessels et al. 2019; Gourdji et al. 2019). It may prove to be a generic property of FRB spectra. On the other hand, narrow burst emission only from a few Galactic neutron stars has been observed to show such bandedness that cannot be explained by scintillation (Hankins et al. 2016; Pearlman et al. 2018; Maan et al. 2019).

\subsection{M33 and M31 halos}

The sky location of FRB 191108 is spatially separated by $1.20 \pm 0.05^{\circ}$ and $13.90 \pm 0.04^{\circ}$ from Local Group galaxies M33 and M31, respectively. As M33 is located at a distance of $840 \mathrm{kpc}$ from the Milky Way, this translates to an impact parameter of $18 \mathrm{kpc}$ to the M33 core. M31 is approximately $770 \mathrm{kpc}$ away, meaning FRB 191108 came within roughly $185 \mathrm{kpc}$ of Andromeda. Since they are relatively nearby, the circumgalactic medium (CGM) around the two galaxies, as well as the baryonic bridge between them, subtend a large angular size. We therefore expect the FRB to have traveled through both galaxies' CGM. Below we consider how these media might have contributed detectable propagation effects to the pulse signature of FRB 191108.

\subsubsection{Local Group DM contribution}

Prochaska \& Zheng (2019) model the CGM of M31, which is large enough to engulf the CGM of M33, as it extends $\sim 30^{\circ}$. They use a modified Navarro-Frenk-White (NFW) profile and assume $M_{\text {halo }}^{\mathrm{M} 31} \approx 1.5 \times 10^{12} \mathrm{M}_{\odot}$ and $M_{\text {halo }}^{\mathrm{M} 33} \approx 5 \times$ $10^{11} \mathrm{M}_{\odot}$. The authors also consider a 'Local Group Medium (LGM)', which models the total intra-group plasma. Using Fig. 9 in that paper, FRB 191108 would have an additional $\sim 40-60 \mathrm{pc} \mathrm{cm}^{-3}$ imparted by the halos of M33 and M31.

The hot gas in the Milky Way halo is also expected to contribute to the DMs of extragalactic objects. Prochaska \& Zheng (2019) estimate a typical contribution of 50$80 \mathrm{pc} \mathrm{cm}^{-3}$. Yamasaki \& Totani (2020) use recent diffuse $\mathrm{X}$-ray observations to model the halo DM, and account for the apparent directional dependence of emission measure (EM). The authors include a hot disk-like halo component as well as the standard spherically symmetric halo to calculate $\mathrm{DM}_{\text {halo }}$ as a function of Galactic longitude and latitude. Using their analytic prescription, we estimate the Milky Way halo contribution to be $30 \pm 20 \mathrm{pc} \mathrm{cm}^{-3}$ in the direction of FRB 191108. Keating \& Pen (2020) find a broader range of allowed values for the Galactic halo DM contribution than previous studies, but also favour smaller values. Combining the estimates of DM from the Milky Way ISM and halo, along with the plasma surrounding M33 and M31, the DM of FRB 191108 beyond the Local Group could be $380-480 \mathrm{pc} \mathrm{cm}^{-3}$.

We use the modelled DM/redshift relation from Petroff et al. (2019), which is consistent with the empirical 'Macquart relation' (Macquart et al. 2020),

$\mathrm{DM}_{\mathrm{IGM}} \approx 930 z \mathrm{pc} \mathrm{cm}^{-3}$

and subtracting off the expected Milky Way and Local Group DM contribution, the implied redshift upper limit on the source is 0.52 . If the $\mathrm{DM}_{\mathrm{IGM}} / z$ relation is reliable, this is a conservative upper limit because it assumes there is zero host-galaxy DM contribution. In the case of FRB 191108, if the Faraday rotation is caused by plasma in the host galaxy, there could be non-negligible dispersion in the same medium and the true host-galaxy redshift would be considerably lower than 0.52 .

ASKAP has also found an FRB that appears to pass through an intervening halo, coming within $\sim 30 \mathrm{kpc}$ of a massive foreground galaxy (Prochaska et al. 2019). This allowed the authors to place constraints on the net magnetization and turbulence in the foreground galaxy halo, due to the relatively low RM and dearth of scattering in FRB 181112.

In our case, the high RM of FRB 191108 does not set a strong upper-limit on the halo magnetic field along the line of sight. Instead we suggest using the large number of polarised extragalactic objects behind M31 and M33 to constrain their CGM (see Fig. 6).

\subsubsection{CGM scattering \& scintillation}

We searched for evidence of scattering in both the pulse profile and the frequency spectrum of FRB 191108. As shown in Sect. 3.3, no temporal scattering was found $\gtrsim 80 \mu \mathrm{s}$. In the frequency spectrum, we find structure on two scales: $\sim 25 \%$ modulations at $40 \mathrm{MHz}$ and $\sim 5 \%$ modulations with a decorrelation bandwidth of 1-2 MHz. For comparison, the NE2001 predicts Galactic diffractive scintillation with a correlation bandwidth of $\approx 1.8 \mathrm{MHz}$ in the FRB direction (Cordes \& Lazio 2002). The $40 \mathrm{MHz}$ structure could either be intrinsic to the source or due to scintillation beyond the Galaxy.

Our data are sensitive to frequency-domain scintillations with corresponding timescale in the range $1 \mathrm{~ns} \lesssim \tau \lesssim 500 \mathrm{~ns}$, set by our $300 \mathrm{MHz}$ band and $0.19 \mathrm{kHz}$ channel width $(\tau \approx 1 / 2 \pi \Delta v)$. To determine the spectral-scale and intensity of scintillations, we compute the autocorrelation function (ACF) of the FRB frequency spectrum and fit it with a Lorentzian function (Lorimer \& Kramer 2004), finding a de-correlation bandwidth of $\Delta v \sim 40 \mathrm{MHz}$, shown in Fig. 5. This appears to be dominated by the patches of increased brightness around $1370 \mathrm{MHz}$ and $1500 \mathrm{MHz}$, which are approximately as wide as the best-fit de-correlation bandwidth. This is more than an order of magnitude larger than the expected Galactic scintillation bandwidth in the 
FRB direction. To search for Galactic scintillation, we removed the frequency modulation on scales above $20 \mathrm{MHz}$ by subtracting a tenth-order polynomial fit from the data, allowing us to look for correlations at smaller $\Delta v$. We found significant structure with a correlation scale of a few $\mathrm{MHz}$ at the level of $5 \%$ intensity modulation.

For a point-like source, diffractive scintillation leads to $100 \%$ modulations of the signal, which we do not see in the 1-2 MHz structure. The level of modulation would be attenuated if an earlier screen has scattered the FRB, leading to angular broadening. For a source with size $\theta_{\mathrm{sc}}$, a scattering screen with a diffractive scale $\theta_{\mathrm{d}}$ leads to a decrease in the modulation RMS of roughly $\theta_{\mathrm{d}} / \theta_{\mathrm{sc}}$. As the intrinsic angular size of the FRB is very small, the most natural place for this angular broadening is the circumgalactic medium of M33 and/or M31. For a characteristic Galactic diffractive scale of $\theta_{\mathrm{d}} \approx 0.1 \mu$ as (Walker 1998), we should expect a $\sim 2 \mu$ as source size for diffractive scintillation to be attenuated to the $\sim 5 \%$ levels.

A $2 \mu$ as angular broadening at M33 also naturally explains the $40 \mathrm{MHz}$-scale structure. A decorrelation bandwidth of $40 \mathrm{MHz}$ corresponds to $\tau \approx 4 \mathrm{~ns}$. For a scattering screen at $d_{\mathrm{M} 33}=840 \mathrm{kpc}$, the corresponding angular broadening scale is $\theta_{\mathrm{sc}} \sim 2 \mu \mathrm{as}-$ the required value. Therefore, scattering in the halo of M33 parsimoniously accounts for both the suppression of Galactic scintillation as well as the broader $40 \mathrm{MHz}$-scale features. It however raises a fresh question as to why the the $40 \mathrm{MHz}$ spectral structure itself, being diffractive in natures, is not observed to be fully modulated. We supply two plausible explanations: (a) We may not be observing a sufficient number of scintels within our bandwidth to measure the total level of modulation. (b) Alternatively, the M33 scintillation could itself be quenched by a scattering in the IGM or CGM of an intervening galaxy. The diffractive scale for the M33 screen in our model is $\theta_{\mathrm{d}} \sim 0.03 \mu \mathrm{as}$. Quenching of fully modulated variations by scattering in intervening CGM (unrelated to M31 \& M33) and/or IGM scintillation to the $25 \%$ level requires angular broadening at the $0.1 \mu$ as scale which is within theoretical expectations (Koay \& Macquart 2015; Vedantham \& Phinney 2019).

We can derive preliminary constraints on the halo-gas parameters by recognising that the diffractive scale $r_{\mathrm{d}}=$ $\lambda /\left(2 \pi \theta_{\mathrm{sc}}\right) \sim 10^{11.5} \mathrm{~cm}$, denotes the transverse extent over which the rms phase variation is unity. Using Coles et al. (1987, their equation 4), for Kolmogovov turbulence, this corresponds to a scattering measure of $\sim 10^{1.3} \mathrm{~cm}^{-17 / 3}$. If the total length through the turbulence is $L \mathrm{~cm}$ and the outer scale of turbulence is $L_{0} \mathrm{~cm}$, then using Macquart \& Koay (2013, their equation 18), the dispersion in the electron density is $\left\langle\delta n_{e}^{2}\right\rangle \sim 10^{2} L_{0}^{2 / 3} / L$. If we further assume that the rms variation in density is equal to the mean density, then we get

$n_{e}=\left\langle\delta n_{e}^{2}\right\rangle^{1 / 2} \sim 10^{-2}\left(\frac{L_{0}}{\mathrm{pc}}\right)^{1 / 3}\left(\frac{L}{\mathrm{pc}}\right)^{-1 / 2} \mathrm{~cm}^{-3}$.

The density implied by equation 4 is too large to be attributed to the virialized $10^{6} \mathrm{~K}$ circumgalactic gas associated with M33. For example, if we assume that the turbulence is driven by galactic outflows on the scale of $L_{0} \sim 10 \mathrm{kpc}$, then the implied density is $n_{e} \sim 7 \times 10^{-4}(L / 100 \mathrm{kpc})^{-1 / 2}$, which is

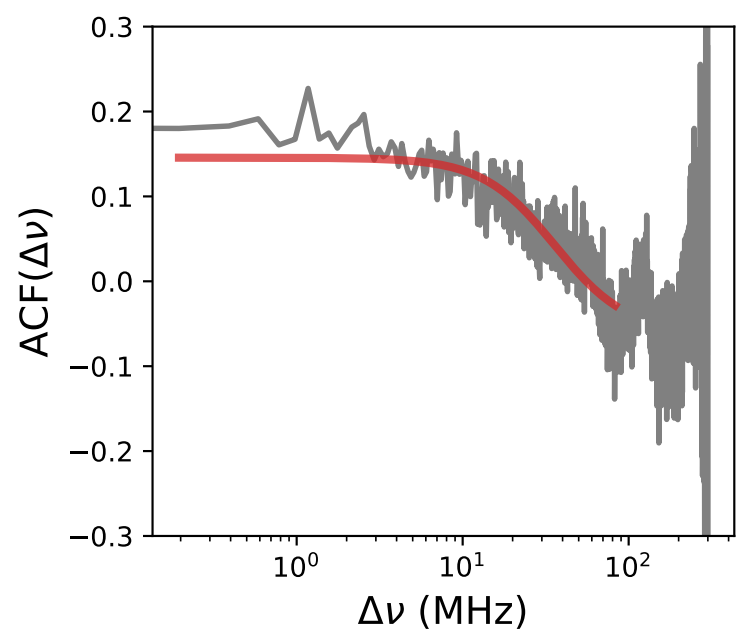

Figure 5: The autocorrelation function of the FRB spectrum, with a best-fit Lorenzian overplotted in red whose de-correlation bandwidth is $40 \mathrm{MHz}$.

two to three orders of magnitude larger than the expected circumgalactic density of M31 and M33 respectively.

Contrary to simple physical models of virialisation in massive dark matter halos, absorption studies have found that most quasars that pass within $\sim 150 \mathrm{kpc}$ of a foreground galaxy indicate the existence of cool $\left(10^{4} \mathrm{~K}\right)$ gas embedded in a hot $\left(10^{6} \mathrm{~K}\right)$ CGM (Prochaska et al. 2014). It has been argued that gas in these environments is prone to fragmentation, leading to a 'cloudlet' model of the CGM in which sub-parsec cold gas clumps are distributed throughout the hot background medium (McCourt et al. 2018). Following the suggestion of Vedantham \& Phinney (2019), we next consider scattering in such cool clumps in the CGM of M31. If the clumps form from cooling instabilities as suggested by McCourt et al. (2018), they will have a density of $\sim 10^{-3} \mathrm{~cm}^{-3}$, and a length-scale of about $20 \mathrm{pc}$ which we take to be the outer scale of turbulence. The path length through the cool clumps is given by the virial radius (200 kpc for M31) times the volume fraction, $f_{V}$. The density constrain from equation 4 for this scenario is $n_{e} \sim 10^{-2.5}\left(f_{V} / 10^{-3}\right)^{-1 / 2} \mathrm{~cm}^{-3}$, which is comparable to the anticipated value.

In summary, the scattering constrain is roughly consistent with expectations from tiny $10^{4} \mathrm{~K}$ clumps formed via cooling instabilities in the circumgalactic medium of M31. We note two caveats here, however. The sight line to the FRB passes close to a neutral gas bridge connecting M31 and M33 (McConnachie et al. 2010). As such, it is unclear if the scattering constraint is probing the specific conditions in neutral bridges or in the general circumgalactic medium. Secondly, with such few scintles we cannot know with certainty if the $\sim 40 \mathrm{MHz}$ decorrelation bandwidth corresponds to scattering or if it is intrinsic to the burst. Future observations of FRBs intersecting the Local Group medium will help answer these questions, including from CHIME where the decorrelation bandwidth would be a $\sim 30$ times narrower. 
Table 1. FRB 191108 parameters. $\dagger$ are values that have been optimised post real-time detection, chosen to maximise $\mathrm{S} / \mathrm{N}$ in the the case of width and DM. Our localisation region is an ellipse whose semi-major and semi-minor axes do not correspond to RA and Dec, so we do not quote uncertainty on those values. For the true sky localisation parameterisation, see Sect. 3.2. The width listed here is dominated by intra-channel dispersion smearing, but we set an upper-limit on its intrinsic width at $80 \mu \mathrm{s}$.

\begin{tabular}{|c|c|}
\hline Date & 2019 November 8 \\
\hline $\mathrm{UTC}^{(\mathrm{a})}$ & 19:48:50.471 \\
\hline $\mathrm{MJD}^{(\mathrm{b})}$ & 58795.830818389 \\
\hline RA (J2000) & $01 \mathrm{~h} 33 \mathrm{~m} 47 \mathrm{~s}$ \\
\hline Dec (J2000) & $+31 \mathrm{~d} 51 \mathrm{~m} 30 \mathrm{~s}$ \\
\hline $\mathrm{DM}^{\dagger}$ & $588.1 \pm 0.1 \mathrm{pc} \mathrm{cm}^{-3}$ \\
\hline $\mathrm{RM}$ & $+474 \pm 3 \mathrm{rad} \mathrm{m}^{-2}$ \\
\hline $\mathrm{Width}^{\dagger}(1370 \mathrm{MHz})$ & $340 \pm 20 \mu \mathrm{s}$ \\
\hline Flux density & $27 \mathrm{Jy}$ \\
\hline Fluence & $11 \mathrm{Jy} \mathrm{ms}$ \\
\hline $\mathrm{S} / \mathrm{N}_{\mathrm{det}}$ & 60 \\
\hline $\mathrm{S} / \mathrm{N}_{\mathrm{opt}}^{\dagger}$ & 103 \\
\hline $\mathrm{DM}_{\mathrm{MW}}(\mathrm{YMW} 16 / \mathrm{NE} 2001)$ & $43 / 52 \mathrm{pc} \mathrm{cm}^{-3}$ \\
\hline $\mathrm{RM}_{\mathrm{MW}}$ & $-50 \mathrm{rad} \mathrm{m}^{-2}$ \\
\hline$z_{\max }$ & 0.52 \\
\hline
\end{tabular}
(a) At $1370 \mathrm{MHz}$.
(b) At the solar system barycenter after removal of the DM delay.

\section{DISCUSSION}

\subsection{Rotation measure origin}

The observed RM of an FRB can be broken down into several components between the observer and source,

$$
\mathrm{RM}_{\mathrm{obs}}=\mathrm{RM}_{\mathrm{MW}}+\mathrm{RM}_{\mathrm{IGM}}+\mathrm{RM}_{\text {host }} \text {, }
$$

where $\mathrm{RM}_{\mathrm{MW}}$ is the foreground RM from the Galaxy, $\mathrm{RM}_{\mathrm{IGM}}$ is from the intergalactic medium, and $\mathrm{RM}_{\text {host }}$ comes from the host galaxy ISM and the region near the FRB progenitor. In the case of FRB 191108, we might also include $\mathrm{RM}_{\mathrm{LG}}$, the contribution from the Local Group. This is the contribution of the galactic halos of M33 (Triangulum) and M31 (Andromeda), and the broader shared plasma linking the two nearby galaxies with the Milky Way. The expected Milky Way foreground is $\mathrm{RM}_{\mathrm{MW}} \approx-50 \mathrm{rad} \mathrm{m}^{-2}$ (Oppermann et al. 2015). Fig. 6 provides an idea of the spatial scatter of this value. Our observed $\mathrm{RM}_{\mathrm{obs}}=+474 \pm 3 \mathrm{rad} \mathrm{m}^{-2}$ thus translates to an estimated extragalactic contribution of approximately $525 \mathrm{rad} \mathrm{m}^{-2}$, which could be up to a couple of times larger in the host-galaxy frame due to cosmological redshift.

Such a large extragalactic RM is not expected from the IGM, as it would require ordered $\mu \mathrm{G}$ magnetic fields over gigaparsec scales to achieve $10^{2-3} \mathrm{rad} \mathrm{m}^{-2}$ for typical FRB redshifts. No intergalactic magnetic fields have been detected, but they are expected to be roughly $\mathrm{nG}$ in strength (Michilli et al. 2018).

We consider the possibility that the ionised material surrounding M33/M31 could contribute all the required magnetised plasma to account for the RM of the FRB, but do not find this compelling for the following reason.
By taking the catalogue of 41632 extragalactic RMs from Oppermann et al. (2012), we identify 93 objects that pass within $5^{\circ}$ of M33, roughly the angular radius of the expected $75 \mathrm{kpc}$ halo. $93 \%$ of these sources have RMs between -15 and $-90 \mathrm{rad} \mathrm{m}^{-2}$ - probably dominated by the Milky Way foreground like most polarised extragalactic sources - and none is larger in magnitude than $100 \mathrm{rad} \mathrm{m}^{-2}$. In Fig. 6 we plot the distribution of extragalactic RMs near the Local Group on the sky to demonstrate the extent to which FRB 191108 is an outlier.

We have also looked at polarised extragalactic sources closer to the FRB sight line in the Apertif imaging data, which has more sources per solid angle than the NVSS RM catalogue (Hess et al. 2021). We find a picture consistent with the Oppermann map (Oppermann et al. 2015), in that the distribution of RMs clusters around $-50 \mathrm{rad} \mathrm{m}^{-2}$ and no point source has an RM as large at FRB 191108. One source is within $\sim 30$ arcminutes of the FRB's best-fit position and likely also intersects the material bridge connecting M33 and M31. Its $\mathrm{RM}$ is $-72 \mathrm{rad} \mathrm{m}^{-2}$, consistent with the values of extragalactic RMs in the surrounding $\sim 10^{\circ} \times 10^{\circ}$.

Therefore, unless the FRB has a very unusual sightline and travels through a dense magnetoionic region in the M33/M31 halo with the opposite magnetic field sign, the absence of strong Faraday rotation in other extragalactic polarised sources behind M33 suggests the FRB RM is imparted elsewhere. The dataset plotted in Fig. 6 and the polarised Apertif imaging data could still be a useful probe of CGM magnetic fields in its own right: the black points in the left panel that have a low impact parameter with M31 show a small gradient such that their amplitude increases towards smaller angular separations. Whether this is due to structure in the Galactic foreground Faraday field or in the M31 halo could be teased out with a Galactic DM map and we leave it to future work to disentangle these effects.

Given we do not expect the large RM of the FRB to be dominated by the Milky Way, M33, or the IGM, it is likely that the magnetised plasma is in the host galaxy. Using the estimated maximum redshift implied by the extragalactic $\mathrm{DM}$, of $z \approx 0.52$, and noting that the local RM will be a factor of $(1+z)^{2}$ larger than the observed RM due to cosmological redshift, $\mathrm{RM}_{\text {host }}$ could be of order $10^{3} \mathrm{rad} \mathrm{m}^{-2}$. Even if the host galaxy contributes significantly to the extragalactic DM and the FRB is much closer than the redshift implied by Eq. 3, the RM would still be much larger than that expected from the ISM of a Milky Way-like galaxy, unless observed very close to edge-on.

FRBs are now known to be located in a range of environments spanning different galaxy types. While there exist examples of polarised FRBs without significant Faraday Rotation (Ravi et al. 2016; Petroff et al. 2017), now including a repeater (Fonseca et al. 2020), several sources appear to pass through regions of highly-magnetised plasma, which may be directly linked to the FRB progenitor itself (e.g. young supernova remnant). Alternatively, FRBs may just be preferentially born in environments that have an abundance of sightlines that intersect, say, HII regions. The first was FRB 110523, which was detected with the Green Bank Telescope. It had an RM of $-186 \mathrm{rad} \mathrm{m}^{-2}$. Like the Apertifdiscovered FRB 191108, this is larger than expected from the Milky Way and the IGM (Masui et al. 2015). The authors argued that its high RM and scattering properties suggested a 
dense magnetised environment local to the source. The FRB with the highest published DM, FRB 160102, had an RM of $-220 \mathrm{rad} \mathrm{m}^{-2}$ (Caleb et al. 2018); its local RM could be as large as $-2400 \mathrm{rad} \mathrm{m}^{-2}$ if a significant portion of the DM comes from the IGM. During Breakthrough Listen observations on the Parkes telescope, FRB 180301 was detected and full-polarisation data was preserved (Price et al. 2019). They report an RM of $-3163 \pm 20 \mathrm{rad} \mathrm{m}^{-2}$, although the patchiness of their frequency spectrum causes the authors to question their Faraday rotation fit. CHIME has found a repeating FRB whose RM exceeds the Galactic foreground by two orders of magnitude, with $\mathrm{RM}=-499.8 \pm 0.7 \mathrm{rad} \mathrm{m}^{-2}$ (Fonseca et al. 2020). Finally, FRB 121102 has an RM of $\sim 10^{5} \mathrm{rad} \mathrm{m}^{-2}$ and is spatially coincident with a bright, compact radio source (Michilli et al. 2018). This is larger than even the Galactic center magnetar, PSR J1745-2900, with RM $\sim 7 \times$ $10^{4} \mathrm{rad} \mathrm{m}^{-2}$ (Eatough et al. 2013). Both FRB 121102 and PSR J1745-2900 have been seen to exhibit significant RM variation over month to year timescales (Desvignes et al. 2018).

The analogy between FRB 121102 and the Galactic center magnetar may extend beyond just phenomenological similarities. If the persistent radio source coincident with FRB 121102 is similar to a low-luminosity active galactic nucleus (LLAGN), then that system may be another example of a circumnuclear magnetar, a scenario that has been proposed as a progenitor theory of FRBs (Pen \& Connor 2015). Alternatively, the radio nebula could correspond to a supernova remnant, magnetar wind nebula, or HII region. Such local environments have been invoked as a way to provide local RM, DM, and scattering (Connor et al. 2016a; Piro 2016; Murase et al. 2016; Piro \& Gaensler 2018; Margalit \& Metzger 2018; Straal et al. 2020). In each of these cases, it is difficult to predict the distribution of observed RMs, but it is likely that the distribution would be broad. For example, in the circumnuclear magnetar model, the FRB RM is a strong function of its distance from the massive black hole. In young magnetar or supernova remnant models, the RM is expected to change with time, and the value depends on when in the progenitor life cycle the FRB was observed. Thus, moderately large RMs like those of FRB 191108, FRB 110523 (Masui et al. 2015), and FRB 160102 (Caleb et al. 2018) may come from a similar environment to FRB 121102.

\subsection{Repetition constraints}

Given the extreme local environment of FRB 121102 and its anomalously high repetition rate, it may be asked if frequent repeaters are more likely to live near dense magnetised plasma. CHIME recently discovered a repeating FRB whose $\mathrm{RM}$ is $-499.8 \pm 0.7 \mathrm{rad} \mathrm{m}^{-2}$, which is roughly two orders of magnitude larger than the expected Milky Way contribution in that direction (Fonseca et al. 2020). But Fonseca et al. (2020) also report a repeater with $\mathrm{RM}=-20 \pm 1 \mathrm{rad} \mathrm{m}^{-2}$, and most of the $\mathrm{RM}=-114.6 \pm 0.6 \mathrm{rad} \mathrm{m}^{-2}$ from another CHIME repeating source, FRB 180916.J0158+65, is thought to be from the Milky Way (CHIME/FRB Collaboration et al. 2019c).

We observed the field of FRB 191108 for 120 hours between July 2019 and December 2019 with Apertif, but had no repeat detections. Apertif has detected and studied other repeating FRBs (Oostrum et al. 2020). Assuming repeti-
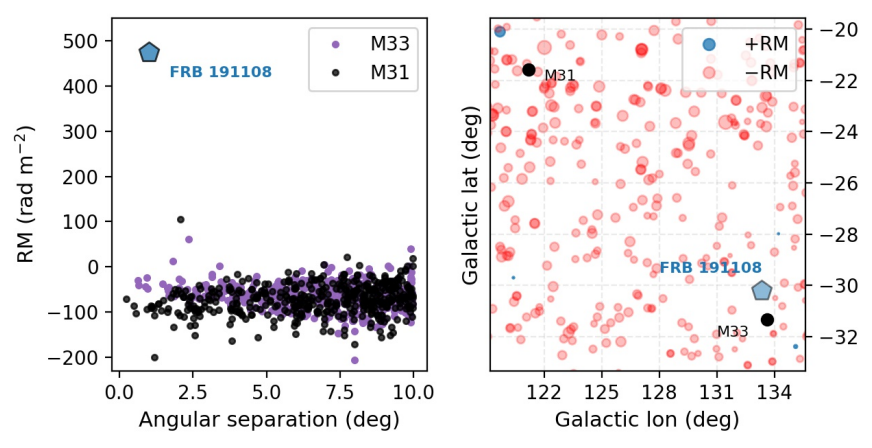

Figure 6: The RMs of extragalactic sources in the direction of the Local Group galaxies M33 and M31. The left panel shows RM vs. angular separation for both M33 (purple) and M31 (black), as well as the FRB which is an outlier both in amplitude and sign. The right panel shows extragalactic sources, where the area of the marker is proportional to $|\mathrm{RM}|$ and the colour encodes its sign. For size reference, the FRB $|\mathrm{RM}|=+474 \mathrm{rad} \mathrm{m}^{-2}$.

tion statistics described by a homogeneous Poisson process, our non-detection provides a $3 \sigma$ upper-limit on the repeat rate of $3 \times 10^{-2}$ per hour. We caution, however, that the assumption of stationarity is known to not be valid for some FRBs, which show time-variability in their repetition rate (Spitler et al. 2016; Oppermann et al. 2018; Gourdji et al. 2019) thereby increasing the probability of seeing zero repeat bursts during follow up (Connor et al. 2016b).

We plan to continue follow-up efforts on the same field, which we can do commensally with our full-FoV blind FRB search. The source is currently localised to an ellipse with semi-minor and semi-major axes of $2.5^{\prime \prime}$ and $3.5^{\prime}$, respectively, as described in Sect. 3.2. If we detect FRB 191108 again at a different hour angle than the initial detection, we will have several arcsecond localisation in both directions, because the TABs rotate as a function of parallactic angle.

\section{CONCLUSIONS}

We have reported the detection of a bright, highly Faraday rotated FRB in the direction of Local Group galaxy M33 using Apertif. By combining multibeam and interferometric information we were able to localise FRB 191108 to a narrow ellipse with radii of $2.5^{\prime \prime}$ and $3.5^{\prime}$. The impact parameter with M33 is just $18 \mathrm{kpc}$, roughly the diameter of that galaxy's disk. The shared plasma in the halos of M33 and M31 likely contributed to the DM of the FRB, but not to its scattering, Faraday rotation, or scintillation. Still, the $\mathrm{RM}$ of $+474 \pm 3 \mathrm{rad} \mathrm{m}^{-2}$ is one of the largest of any published value and is an order of magnitude larger than the expected contribution from the Milky Way, the IGM, and these halos. The most plausible location of the magnetised plasma is therefore a dense region near the FRB-emitting source itself.

\section{ACKNOWLEDGEMENTS}

We thank Dan Stinebring, Jason Hessels, and Monica Relano Pastor for helpful conversations. This research was supported by the European Research Council under the Eu- 
ropean Union's Seventh Framework Programme (FP/20072013)/ERC Grant Agreement No. 617199 ('ALERT'), and by Vici research programme 'ARGO' with project number 639.043.815, financed by the Dutch Research Council (NWO). Instrumentation development was supported by NWO (grant 614.061.613 'ARTS') and the Netherlands Research School for Astronomy ('NOVA4-ARTS' and 'NOVANW3'). EP acknowledges funding from an NWO Veni Fellowship. AS and DV acknowledge support from the Netherlands eScience Center (NLeSC) under grant ASDI.15.406. SMS acknowledges support from the Netherlands Research School for Astronomy (NOVA4-ARTS), and was supported by the National Aeronautics and Space Administration (NASA) under grant number NNX17AL74G issued through the NNH16ZDA001N Astrophysics Data Analysis Program (ADAP). EAKA is supported by the WISE research programme, which is financed by NWO.

\section{REFERENCES}

Adams E. A. K., van Leeuwen J., 2019, Nature Astronomy, 3, 188 Bannister K. W., et al., 2017, ApJ, 841, L12

Bannister K. W., et al., 2019, Science, 365, 565

Bassa C. G., et al., 2017, ApJ, 843, L8

CHIME/FRB Collaboration et al., 2019a, Nature, 566, 230

CHIME/FRB Collaboration et al., 2019b, Nature, 566, 235

CHIME/FRB Collaboration et al., 2019c, ApJ, 885, L24

Caleb M., et al., 2018, MNRAS, 478, 2046

Chatterjee S., et al., 2017, Nature, 541, 58

Cho H., et al., 2020, ApJ, 891, L38

Chomiuk L., Wilcots E. M., 2009, ApJ, 703, 370

Coles W. A., Frehlich R. G., Rickett B. J., Codona J. L., 1987, ApJ, 315, 666

Connor L., 2019, MNRAS, 487, 5753

Connor L., van Leeuwen J., 2018, AJ, 156, 256

Connor L., Sievers J., Pen U.-L., 2016a, MNRAS, 458, L19

Connor L., Pen U.-L., Oppermann N., 2016b, MNRAS, 458, L89

Cordes J. M., Chatterjee S., 2019, ARA\&A, 57, 417

Cordes J. M., Lazio T. J. W., 2002, arXiv e-prints, pp astro$\mathrm{ph} / 0207156$

Desvignes G., et al., 2018, ApJ, 852, L12

Eatough R. P., et al., 2013, Nature, 501, 391

Eftekhari T., Berger E., 2017, ApJ, 849, 162

Eftekhari T., Berger E., Williams P. K. G., Blanchard P. K., 2018, ApJ, 860, 73

Eichler D., 2017, ApJ, 850, 159

Farah W., et al., 2018, MNRAS, 478, 1209

Fonseca E., et al., 2020, ApJ, 891, L6

Gajjar V., et al., 2018, ApJ, 863, 2

Gourdji K., Michilli D., Spitler L. G., Hessels J. W. T., Seymour A., Cordes J. M., Chatterjee S., 2019, ApJ, 877, L19

Hankins T. H., Eilek J. A., Jones G., 2016, ApJ, 833, 47

Hess K., et al., 2020.

Hess K. M., Apertif Contributors Apertif Builders 2021, MNRAS

Hessels J. W. T., et al., 2019, ApJ, 876, L23

Keating L. C., Pen U.-L., 2020, MNRAS, 496, L106

Koay J. Y., Macquart J. P., 2015, MNRAS, 446, 2370

Kumar P., et al., 2019, The Astrophysical Journal, 887, L30

Lorimer D. R., Kramer M., 2004, Handbook of Pulsar Astronomy. Vol. 4

Lorimer D. R., Bailes M., McLaughlin M. A., Narkevic D. J., Crawford F., 2007, Science, 318, 777

Maan Y., Joshi B. C., Surnis M. P., Bagchi M., Manoharan P. K., 2019, ApJL, 882, L9

Macquart J.-P., Koay J. Y., 2013, ApJ, 776, 125
Macquart J. P., et al., 2020, Nature, 581, 391

Marcote B., et al., 2017, ApJ, 834, L8

Marcote B., et al., 2020, Nature, 577, 190

Margalit B., Metzger B. D., 2018, ApJ, 868, L4

Masui K., et al., 2015, Nature, 528, 523

McConnachie A. W., et al., 2009, Nature, 461, 66

McConnachie A. W., Ferguson A. M. N., Irwin M. J., Dubinski J., Widrow L. M., Dotter A., Ibata R., Lewis G. F., 2010, ApJ, 723, 1038

McCourt M., Oh S. P., O'Leary R., Madigan A.-M., 2018, MNRAS, 473, 5407

McQuinn M., 2014, ApJ, 780, L33

Michilli D., et al., 2018, Nature, 553, 182

Muñoz J. B., Kovetz E. D., Dai L., Kamionkowski M., 2016, Phys. Rev. Lett., 117, 091301

Murase K., Kashiyama K., Mészáros P., 2016, MNRAS, 461, 1498

Oosterloo T., Verheijen M., van Cappellen W., 2010, in "ISKAF2010 Science Meeting", Van Leeuwen, Morganti, Serra (Eds.). (arXiv: 1007.5141)

Oostrum L. C., 2020a, DARC: Data Analysis of Real-time Candidates, doi:10.5281/zenodo.3784870, https://doi.org/10. $5281 /$ zenodo. 3784870

Oostrum L. C., 2020b, PhD thesis, University of Amsterdam

Oostrum L. C., et al., 2020, A\&A, 635, A61

Oppermann N., et al., 2012, A\&A, 542, A93

Oppermann N., et al., 2015, A\&A, 575, A118

Oppermann N., Yu H.-R., Pen U.-L., 2018, MNRAS, 475, 5109

Paladini R., DeZotti G., Noriega-Crespo A., Carey S. J., 2009, The Astrophysical Journal, 702, 1036

Pearlman A. B., Majid W. A., Prince T. A., Kocz J., Horiuchi S., 2018, ApJ, 866, 160

Pen U.-L., Connor L., 2015, ApJ, 807, 179

Petroff E., et al., 2016, Publ. Astron. Soc. Australia, 33, e045

Petroff E., et al., 2017, Monthly Notices of the Royal Astronomical Society, 469, 4465

Petroff E., Hessels J. W. T., Lorimer D. R., 2019, A\&ARv, 27, 4

Piro A. L., 2016, ApJ, 824, L32

Piro A. L., Gaensler B. M., 2018, ApJ, 861, 150

Price D. C., et al., 2019, MNRAS, 486, 3636

Prochaska J. X., Zheng Y., 2019, MNRAS, 485, 648

Prochaska J. X., Lau M. W., Hennawi J. F., 2014, ApJ, 796, 140

Prochaska J. X., et al., 2019, Science, 365, aay0073

Ravi V., et al., 2016, Science, 354, 1249

Ravi V., et al., 2019, Nature, 572, 352

Relano M., et al., 2013, Astronomy and Astrophysics, 552

Sclocco A., van Leeuwen J., Bal H. E., van Nieuwpoort R. V., 2016, Astronomy and Computing, 14, 1

Sclocco A., Heldens S., van Werkhoven B., 2020, SoftwareX, 12, 100549

Shannon R. M., et al., 2018, Nature, 562, 386

Spitler L. G., et al., 2014, ApJ, 790, 101

Spitler L. G., et al., 2016, Nature, 531, 202

Straal S. M., Connor L., van Leeuwen J., 2020, A\&A, 634, A105

Tendulkar S. P., et al., 2017, ApJ, 834, L7

Vedantham H. K., Phinney E. S., 2019, MNRAS, 483, 971

Walker M. A., 1998, MNRAS, 294, 307

Walters A., Weltman A., Gaensler B. M., Ma Y.-Z., Witzemann A., 2018, ApJ, 856, 65

Yamasaki S., Totani T., 2020, ApJ, 888, 105

Yao J. M., Manchester R. N., Wang N., 2017, ApJ, 835, 29

York D. G., et al., 2000, AJ, 120, 1579

van Leeuwen J., Kooistra E., Connor L., Maan Y., Oostrum L. C., et al. 2020, A\&A, in prep 